\title{
Temporal differences in microbial composition of Époisses cheese rinds during ripening and storage
}

\author{
F. Irlinger (1) and C. Monnet* (1) \\ Université Paris-Saclay, INRAE, AgroParisTech, UMR SayFood, 78850 Thiverval-Grignon, France
}

\section{ABSTRACT}

Époisses is a protected designation of origin smearripened cheese from the Burgundy region in France. It has an orange color and a strong flavor, both of which are generated by surface microorganisms. The objective of the present study was to investigate the microbial dynamics at the surface of Époisses cheese during ripening and postmanufacturing storage at low temperatures. Rind samples were analyzed by enumeration on agar plates and by $16 \mathrm{~S}$ rRNA gene and internal transcribed spacer amplicon sequencing. During most of the ripening process, the counts of yeasts, which corresponded to the species Debaryomyces hansenii and Geotrichum candidum, were higher than those of the aerobic acid-sensitive bacteria. Debaryomyces hansenii reached a level of about $3 \times 10^{8} \mathrm{cfu} / \mathrm{cm}^{2}$, and its viability strongly decreased in the late stage of ripening and during storage at $4^{\circ} \mathrm{C}$. Two of the inoculated bacterial species, Brevibacterium aurantiacum and Staphylococcus xylosus, did not establish themselves at the cheese surface. At the end of ripening, among the 18 most abundant bacterial species detected by amplicon sequencing, 14 were gram-negative, mainly from genera Psychrobacter, Vibrio, Halomonas, and Mesonia. It was hypothesized that the high moisture level of the Époisses rinds, due the humid atmosphere of the ripening rooms and to the frequent washings of the curds, favored growth of these gram-negative species. These species may be of interest for the development of efficient ripening cultures. In addition, because the orange color of Époisses cheeses could not be attributed to the growth of Brevibacterium, it would be interesting to investigate the type and origin of the pigments that confer color to this cheese.

Key words: smear-ripened cheese, ripening, Époisses, Debaryomyces hansenii, Mesonia ostreae

Received January 4, 2021.

Accepted February 24, 2021

*Corresponding author: christophe.monnet@inrae.fr

\section{INTRODUCTION}

Smear-ripened cheeses are covered by a layer of yeasts and bacteria that have a strong effect on appearance and flavor (Bockelmann, 2011). The yeasts dominate during the early stages of ripening because they are salt- and acid-tolerant, and subsequently increase the $\mathrm{pH}$, thereby favoring the growth of aerobic acid-sensitive bacteria. Ripening takes place at high relative humidity $(>95 \%)$, and the cheeses are washed several times with a dilute saline solution to spread any microcolonies of yeasts or bacteria that have developed on the surface. The beneficial yeasts and bacteria have to be competitive to prevent the growth of undesired microorganisms, and most smear-ripened cheese are inoculated with defined ripening cultures today (Bockelmann et al., 2005).

As for other food products, microbial analyses of smear-ripened cheeses traditionally relied on culturebased techniques, but the advent of culture-independent techniques based on the analysis of nucleic acids extracted from the food matrix was very useful to better characterize the structure of the microbial communities (De Filippis et al., 2018; Jonnala et al., 2018). However, only limited information is available about the microbial dynamics that occur during the manufacturing of smear-ripened cheeses. Such information may help to better understand the ecological relationships between cheese microorganisms and their adaptation to the cheese habitat. For example, population dynamic studies of Gubbeen cheeses revealed a progression of bacteria, with staphylococci dominating the early stages of ripening and Actinobacteria the later stages, and the commercial strains used for smearing the cheese were only present at a very low level early in ripening (Rea et al., 2007). In Tilsit cheeses, biodiversity increased during ripening and Actinobacteria were the most prominent group in the late phase.

The objective of the present study was to evaluate the microbial dynamics at the surface of Époisses, a French protected designation of origin cheese (LEGIFRANCE, 2010), whose production level is approximately 1,300 $\mathrm{t}$ per year (CNIEL, 2020). This cheese is made from whole cow's milk produced by specified dairy breeds in 
the Burgundy region. Époisses cheese can be manufactured from raw or from pasteurized milk and undergoes a dominant lactic type coagulation, whose duration is greater than $16 \mathrm{~h}$. Draining occurs spontaneously, without pressing. Salting is done using dry salt, and ripening time is at least $28 \mathrm{~d}$ at a temperature of $\sim 10$ to $13^{\circ} \mathrm{C}$. During ripening, the cheeses are washed 1 to 3 times per week with washing solutions supplied with increasing amounts of Marc de Bourgogne, a pomace brandy. Epoisses cheese has an orange color and a strong flavor, both generated by surface microorganisms. A 16S rRNA amplicon-sequencing analysis revealed that Époisses rinds contain high levels of species adapted to cold aquatic and saline environments (Dugat-Bony et al., 2016). To better understand the microbial activity involved in the generation of the typical properties of Époisses, as well as to provide information about ripening cultures that are suitable for this cheese variety, it was interesting to monitor the microbial composition during a typical manufacturing run and postmanufacturing storage at low temperatures.

\section{MATERIALS AND METHODS}

\section{Cheese Manufacturing and Sampling}

All the cheeses were taken from the same batch of cheese production of an industrial manufacturing unit in 2017. The factory produced the cheeses from pasteurized milk and in compliance with the Époisses PDO specifications. Several commercial microbial cultures were inoculated during cheesemaking. At the beginning of the manufacturing process, milk was inoculated with a mesophilic lactic starter culture, with the yeasts Debaryomyces hansenii and Geotrichum candidum and with the aerobic ripening bacteria Brevibacterium aurantiacum, Staphylococcus xylosus, and Glutamicibacter arilaitensis. Brevibacterium aurantiacum was also added to the smearing solutions used after d 6 . After ripening $\left(12^{\circ} \mathrm{C}\right)$, the cheeses were packaged and transferred to a cold room at $4^{\circ} \mathrm{C}$. Three different cheeses were analyzed at 8 sampling times (d 6, 9, 21, 24, 28, 33,40 , and 90). The cheeses were cut perpendicular to the surface to produce 2 equivalent parts. One part was used to measure the concentrations of lactose, lactate, free AA, and nonprotein fractions. The upper and lower sections (rinds) of the other part were removed with a knife (thickness $\sim 2-3 \mathrm{~mm}$ ), pooled, and used for cell counts, $\mathrm{pH}$ measurements, and DNA extraction.

\section{Microbiological Analyses}

One gram of cheese rind, sampled as described above, was mixed with $9 \mathrm{~mL}$ of physiological saline solution (9 $\mathrm{g} / \mathrm{L} \mathrm{NaCl}$ ). After dispersion with a mechanical blender (Ultra Turrax model T25; Ika Labortechnik) for $1 \mathrm{~min}$ at 14,000 rpm, 10-fold serial dilutions were performed in physiological saline solution and plated in triplicate on agar plates. After $4 \mathrm{~d}$ of incubation at $25^{\circ} \mathrm{C}$, the aerobic ripening bacteria were counted on brain heart infusion agar (Biokar Diagnostics) supplemented with $50 \mathrm{mg} / \mathrm{L}$ amphotericin (Sigma Aldrich), which inhibits the growth of fungi. The lactic acid bacteria were counted on De Man, Rogosa and Sharpe agar (Biokar diagnostics) supplemented with $50 \mathrm{mg} / \mathrm{L}$ amphotericin after $3 \mathrm{~d}$ of incubation at $30^{\circ} \mathrm{C}$ under anaerobic conditions. The yeasts were counted on yeast extract-glucose-chloramphenicol agar (Biokar Diagnostics) after $3 \mathrm{~d}$ of incubation at $25^{\circ} \mathrm{C}$. Geotrichum candidum and $D$. hansenii could be selectively counted on this medium because they have distinct colony morphotypes.

\section{Biochemical Analyses}

Biochemical analyses were performed for each of the 3 cheeses sampled at the 8 sampling times. Nonprotein nitrogen content of the cheese was measured by the Kjeldahl method (ISO, 2016). The levels of lactate and lactose were assayed using commercially available kits (Biosentec) according to the manufacturer's instructions. Free AA were analyzed by HPLC, as previously described (Castellote et al., 2015). The $\mathrm{pH}$ was measured on the homogenized cheese rinds.

\section{DNA Extraction, 16S rRNA Internal Transcribed Spacer Amplicon Sequencing, and Data Analysis}

We extracted DNA from 3 different cheeses at each of the 8 sampling times, using the bead beating-based protocol detailed in a previous study (Dugat-Bony et al., 2015). Briefly, the cheese rind samples $(\sim 1 \mathrm{~g})$ were diluted in $9 \mathrm{~mL}$ of sodium citrate solution $(20 \mathrm{~g} / \mathrm{L}$ trisodium citrate dihydrate) and homogenized with an Ultra Turrax model T25 mechanical blender at 24,000 rpm for $2 \mathrm{~min}$. A second treatment was performed after 10 min of incubation at room temperature. The mixture was then centrifuged at $6,400 \times g$ for $10 \mathrm{~min}$ at $4^{\circ} \mathrm{C}$, and the supernatant was removed. The casein pellet (containing the microbial cells) was resuspended in 5 $\mathrm{mL}$ of a Triton X-100 aqueous solution $(2.5 \% \mathrm{vol} / \mathrm{vol})$, vigorously shaken, heated in a water bath at $70^{\circ} \mathrm{C}$ for 10 min, centrifuged at $6,400 \times g$ for $10 \mathrm{~min}$ at $4^{\circ} \mathrm{C}$, and rinsed twice with $10 \mathrm{~mL}$ of a physiological saline solution. The pellet was dissolved in a mixture of $270 \mu \mathrm{L}$ of guanidium thiocyanate $(4 M)$ in Tris- $\mathrm{HCl}(\mathrm{pH} 7.5,0.1$ $M)$ and $30 \mu \mathrm{L}$ of sodium lauroyl sarcosinate $(100 \mathrm{~g} / \mathrm{L})$, and transferred to a $2-\mathrm{mL}$ tube containing $250 \mathrm{mg}$ of $0.1-\mathrm{mm}$ diameter zirconium beads and $250 \mathrm{mg}$ of $0.5-$ 
mm diameter zirconium beads (Biospec Products). Proteinase $\mathrm{K}$ treatment, bead beating, phenol-chloroform extraction, RNase treatment, and ethanol precipitation were then performed as previously described (LeclercqPerlat et al., 2013), and the DNA pellet was dissolved in $120 \mu \mathrm{L}$ of Tris EDTA buffer $(10 \mathrm{~m} M$ Tris-HCl, $\mathrm{pH}$ 8.0, $1 \mathrm{~m} M$ EDTA). Purified DNA was quantified with Qubit DNA assay kits on the Qubit 3.0 fluorometer (ThermoFischer Scientific). The PCR amplification of the bacterial V3-V4 region (16S rRNA gene) and of the fungal ribosomal internal transcribed spacer (ITS) 2 region was done with the primer pairs F343/R784 and ITS3/ITS4_HYO1, as previously described (DugatBony et al., 2016). The amplified products were used for Illumina paired-end library preparation and cluster generation, followed by 250 bp paired-end sequencing on an Illumina MiSeq instrument (INRAE, GeT-PlaGE platform). Paired-end reads $(\sim 2 \times 25,000$ sequences $)$ were merged using Flash (Magoč and Salzberg, 2011), and the sequence data were processed using the FROGS pipeline (Escudié et al., 2018), according to the standard operating procedure. Briefly, operational taxonomic units (OTU) were built using Swarm with a maximum number of differences allowed to group together 2 amplicons (aggregation distance parameter) of 3 (Mahé et al., 2014), and OTU that accounted for $<0.05 \%$ of the total set of sequences were discarded. Last, the OTU affiliations were checked using the EzBiocloud database (Kim et al., 2012) for the bacteria and the UNITE database (Nilsson et al., 2019) for the fungi. Alpha-diversity metrics were determined using the Phyloseq package (McMurdie and Holmes, 2013) implemented on the FROGS pipeline. Bacterial OTUsample networks were computed using Gephi software (v. 0.9.1; Bastian et al., 2009). For a better visualization, only edges corresponding to the presence of OTU with a relative abundance $>0.5 \%$ in the samples were represented on the graph. Raw sequence data were deposited at the Sequence Read Archive of the National Center for Biotechnology Information under the accession numbers SAMN17082512 to SAMN17082535 (bioproject PRJNA685310).

\section{RESULTS}

To investigate the microbial dynamics at the surface of Époisses cheeses, samples corresponding to the same manufacturing run of a cheese factory were taken at various ripening stages and during storage at $4^{\circ} \mathrm{C}$. A white covering became visible at the surface of the cheeses sampled at d 21, and a yellow-orange color, typical of this cheese variety, appeared afterward with increasing intensity during storage at $4^{\circ} \mathrm{C}$ (Figure $1 \mathrm{~A}$ ). The $\mathrm{pH}$ of the cheese surface increased from about 4.5
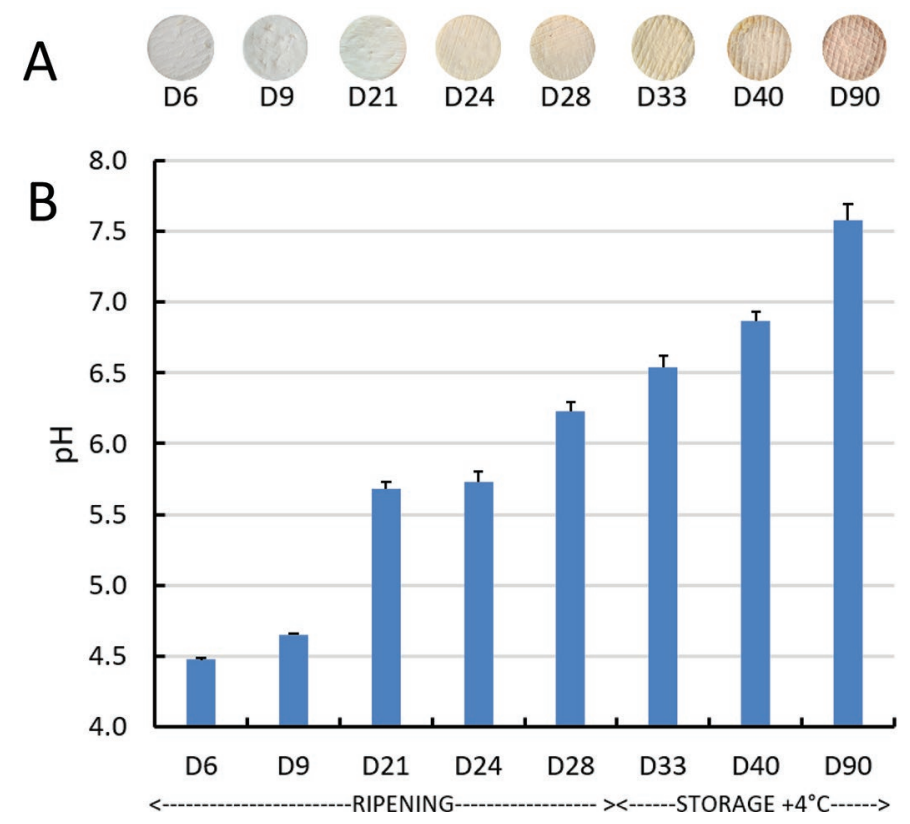

Figure 1. (A) Appearance of the Époisses cheeses during their manufacturing and storage. (B) Changes in the $\mathrm{pH}$ values at the surface of the cheeses. Bars show mean $\pm \mathrm{SD}$ of 3 replicates. The samples D6, D9, D21, D24, D28, D33, D40, and D90 correspond to cheeses sampled from 6 to $90 \mathrm{~d}$ after the beginning of manufacturing.

at $\mathrm{d} 6$ to 6.5 at $\mathrm{d} 33$, and continued to increase after packaging and storage at $4^{\circ} \mathrm{C}$ (Figure 1B). Lactose and lactate were consumed by the cheese microorganisms and were mostly exhausted in the cheeses at the end of ripening (Figure 2). During ripening, the NPN fraction of the cheeses increased, and it continued to increase during storage at $4^{\circ} \mathrm{C}$. The production of NPN results from the degradation of proteins and AA.

\section{Fungal Growth at the Cheese Surface}

At the cheese surface, the yeast $D$. hansenii reached about $3 \times 10^{8} \mathrm{cfu} / \mathrm{cm}^{2}$ at d 21 and 28 , and cell numbers strongly decreased thereafter (Figure 3). Geotrichum candidum was not detected at d 6 and 9 , but this may be due to the fact that it was not possible to distinguish $G$. candidum colonies on the agar plates when this species represented less than about $1 \%$ of $D$. hansenii because of the large colonies formed by the latter that rapidly covered the entire surface of the agar plates. In contrast to D. hansenii, the level of $G$. candidum was approximately constant from d 21 to d 90. The cheese rind contained lactic acid bacteria, whose level slightly decreased during ripening. The level of aerobic bacteria was about $3 \times 10^{4} \mathrm{cfu} / \mathrm{cm}^{2}$ at d 21 and reached $2 \times 10^{8} \mathrm{cfu} / \mathrm{cm}^{2}$ at $\mathrm{d} 33$. This growth is probably the consequence of the increase in the cheese $\mathrm{pH}$ due to the activity of the yeasts, which favors the growth of acid- 


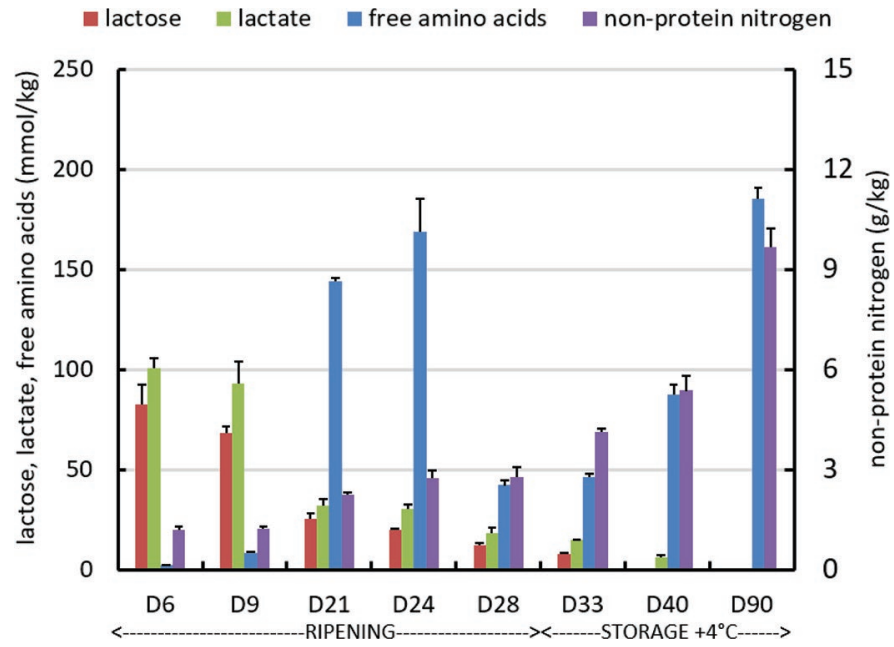

Figure 2. Concentration of lactose, lactate, free AA, and NPN fractions during the manufacturing and storage of Époisses cheeses. Bars show mean $\pm \mathrm{SD}$ of 3 replicates. The samples D6, D9, D21, D24, D28, D33, D40, and D90 correspond to cheeses sampled from 6 to 90 $\mathrm{d}$ after the beginning of manufacturing.

sensitive surface bacteria. Despite the use of orangepigmented $B$. aurantiacum strains as ripening agents, orange colonies were not or almost never observed on the agar plates from cheeses sampled from $\mathrm{d} 6$ to $\mathrm{d}$ $40(<1 \%$ of the colonies enumerated on brain heart infusion agar), and represented only about $2 \%$ of the colonies at d 90.

The ITS amplicon-sequencing analyses revealed a lower level of $G$. candidum sequences in comparison to D. hansenii at the beginning of ripening (Figure 4A).

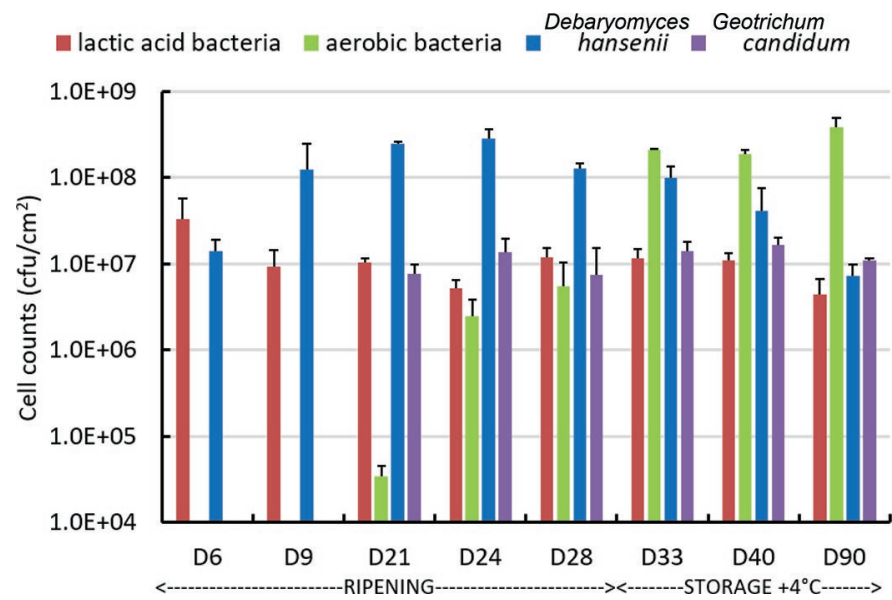

Figure 3. Bacterial and yeast counts at the surface of the Époisses cheeses during their manufacturing and storage. Bars show mean \pm SD of 3 replicates. The samples D6, D9, D21, D24, D28, D33, D40, and D90 correspond to cheeses sampled from 6 to $90 \mathrm{~d}$ after the beginning of manufacturing.
From $\mathrm{d} 21$ to 90 , the changes in the proportion of the ITS sequences of the 2 yeasts was different than what was observed by counting on agar plates (Figure 5). Indeed, after d 21, there was only a slight increase in the proportion of the G. candidum ITS sequences, whereas a large increase was observed for the proportion of $G$. candidum living cells. This may be explained by the large decrease of $D$. hansenii viability that was observed after d 24. Except for G. candidum and D. hansenii, no other fungi were detected in the cheeses (cutoff was set at $0.05 \%$ relative abundance of the ITS sequences).

\section{Bacterial Growth at the Cheese Surface}

The 16S rRNA amplicon-sequencing analyses showed the presence of 2 sequence clusters of lactic acid bacteria (Figure 4B and Supplemental Table S1, https:/ /doi.org/10.15454/LFA0VN/2BXJ3I). The first corresponds to L. lactis and the second (referred to as Lactococcus group chungangensis) to L. chungangensis and L. laudensis. The proportion of the lactic acid bacteria $16 \mathrm{~S}$ rRNA sequences considerably decreased during ripening, especially after $\mathrm{d} 24$, as the consequence of the growth of the aerobic acid-sensitive bacteria. At the late stage of ripening, there was a large increase of $16 \mathrm{~S}$ rRNA sequences corresponding to $G$. arilaitensis, Psychrobacter aquimaris, Psychrobacter group nivimaris, Psychrobacter cibarius, Vibrio litoralis, Halomonas group venusta, and Mesonia ostreae. Among these bacteria, only $G$. arilaitensis was deliberately inoculated as a ripening agent. The other inoculated ripening bacteria, B. aurantiacum and S. xylosus, did not establish themselves at the surface of the cheese. The $16 \mathrm{~S}$ rRNA sequences of $S$. xylosus were not detected $(0.05 \%$ cutoff), and those of $B$. aurantiacum represented less than $0.5 \%$ of the average relative abundance, except in 1 cheese sample at d $6(1.0 \%)$ and in 1 cheese sample at d $90(2.2 \%)$. At d 24, P. aquimaris was the most abundant uninoculated species that grew at the surface of the cheeses and represented between 7 and $13 \%$ of the $16 \mathrm{~S}$ rRNA sequences. The $16 \mathrm{~S}$ rRNA sequences from this species were also abundant in 1 of the cheeses at $d$ 6 and 1 at $d 21$, showing that there were some differences in cheeses sampled at the same ripening time. At the end of ripening at $\mathrm{d} 33$, lactic acid bacteria represented less than $10 \%$ of the $16 \mathrm{~S}$ rRNA sequences, and, in addition to $P$. aquimaris, the most abundant OTU were G. arilaitensis, Psychrobacter group nivimaris, $V$. litoralis, Halomonas group venusta, and M. ostreae. The bacterial composition changed during storage at $4^{\circ} \mathrm{C}$. The proportion of $G$. arilaitensis increased, whereas there was a decrease for $P$. aquimaris, Psychrobacter group nivimaris, V. litoralis, and $M$. ostreae. Eight minor bacterial groups ("other" category in Figure 4B), 
representing an average relative abundance of between 0.05 and $0.3 \%$, were detected by $16 \mathrm{~S}$ rRNA amplicon sequencing: Halomonas titanicae, Halomonas glaciei, Psychrobacter proteolyticus, Psychrobacter namhaensis, Pseudoalteromonas group issachenkonii, Marinomonas polaris, Cobetia group marina, and Sphingomonas group insulae (Supplemental Table S1, https://doi.org/ 10.15454/LFA0VN/2BXJ3I). Shannon and Inverse Simpson $\alpha$-diversity indices showed that the highest bacterial diversity was at d 28 and 33 and decreased during storage at $4^{\circ} \mathrm{C}$ (Supplemental Figure S1, https:/ /doi.org/10.15454/LFA0VN/3L8EYI).
Because a previous study concerning the bacterial composition of Époisses cheese rinds from 3 different factories in 2014 was available (Dugat-Bony et al., 2016), we recovered the corresponding $16 \mathrm{~S}$ rRNA sequencing data (accession number SRP071345 of the Sequence Read Archive of the National Center for Biotechnology Information, https://trace.ncbi.nlm.nih .gov/Traces/sra/?study=SRP071345) and processed them using the bioinformatic pipeline described in the Materials and Methods section. The same protocol for DNA extraction and amplicon sequencing was used in the 2 studies. An OTU-sample network was built to

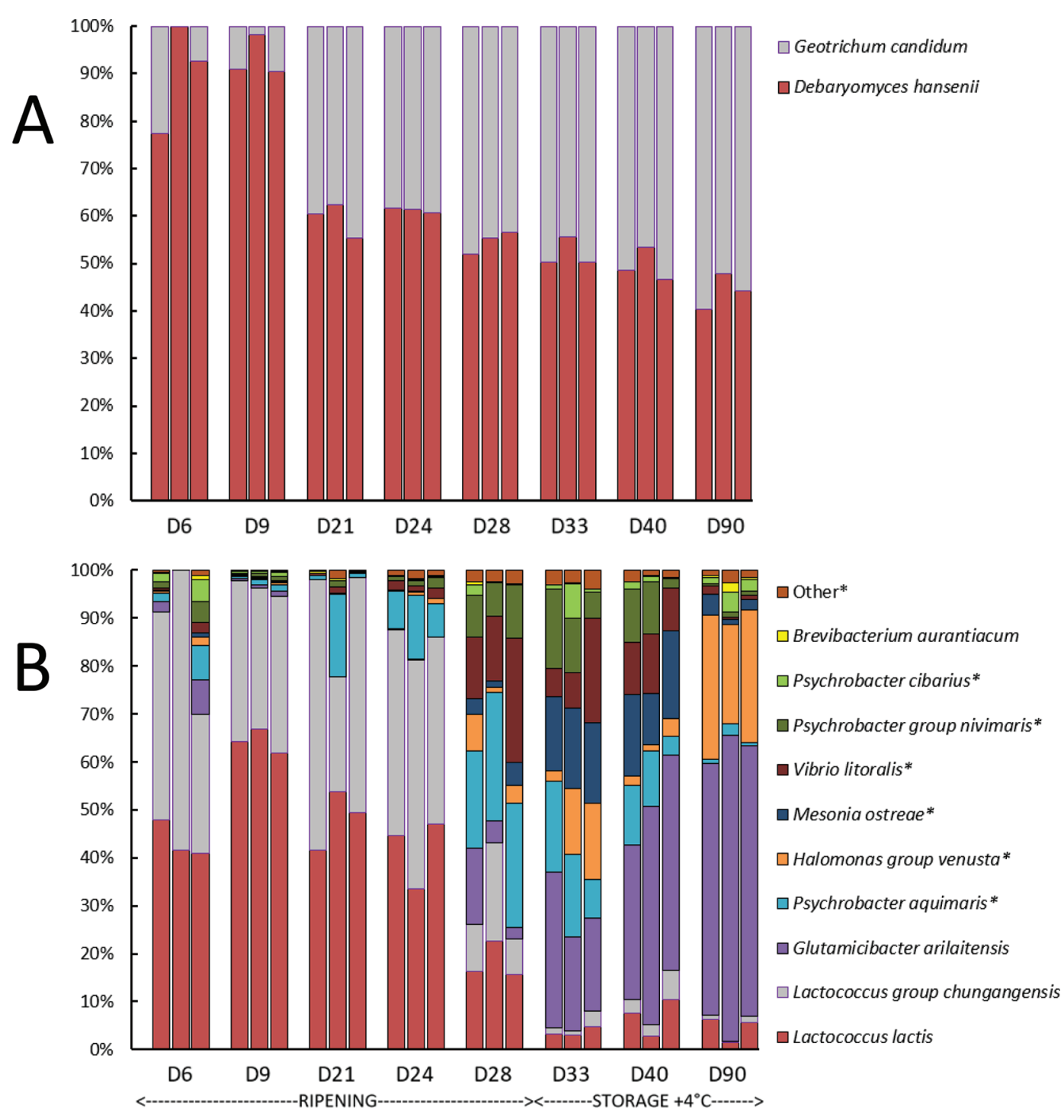

Figure 4. Relative abundances of the fungal (A) and bacterial (B) species at the surface of the Époisses cheeses during their manufacturing and storage. Abundances were estimated by $16 \mathrm{~S}$ rRNA and internal transcribed spacer marker gene analysis. Microorganisms that were not inoculated during cheesemaking are indicated by an asterisk. The "other" category corresponds to the subdominant species Halomonas titanicae, Halomonas glaciei, Psychrobacter proteolyticus, Psychrobacter namhaensis, Pseudoalteromonas group issachenkonii, Marinomonas polaris, Cobetia group marina, and Sphingomonas group insulae. Three separate cheeses were analyzed at each sampling time. The samples D6, D9, D21, D24, D28, D33, D40 and D90 correspond to cheeses sampled from 6 to $90 \mathrm{~d}$ after the beginning of manufacturing. 


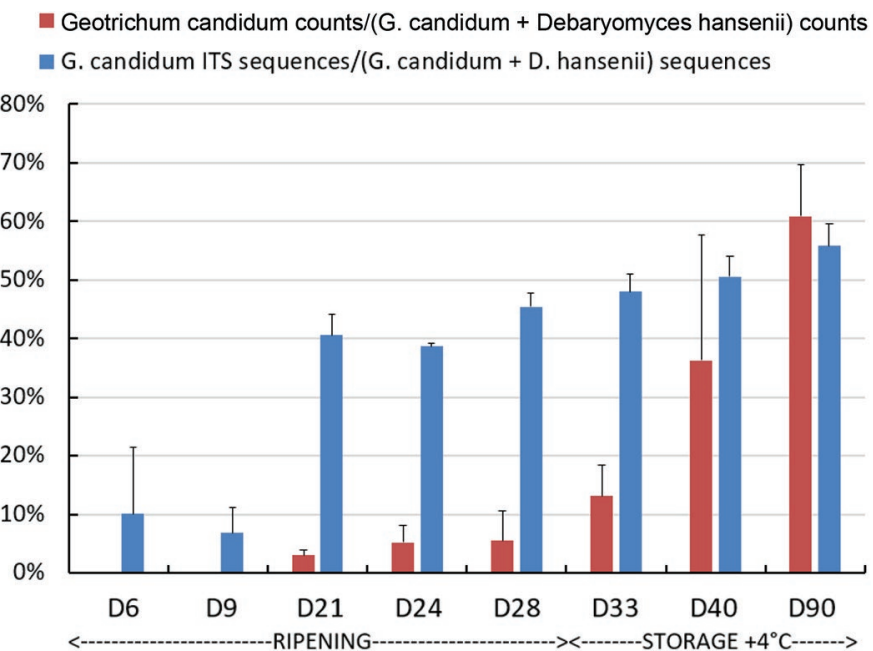

Figure 5. Proportion of Geotrichum candidum among the yeasts at the surface of the Époisses cheeses during their manufacturing and storage. Abundances were estimated by colony counting and by internal transcribed spacer marker gene analysis. The samples D6, D9, D21, D24, D28, D33, D40, and D90 correspond to cheeses sampled from 6 to $90 \mathrm{~d}$ after the beginning of manufacturing.

visualize differences between the samples (Figure 6). The cheese samples that were investigated in the present study were produced by factory 1 . The absence of Brevibacterium and the dominance of gram-negative species such as Psychrobacter are observed in all of the samples. In comparison to the cheeses produced by the factory 1 in 2014, the cheeses produced in 2017 (present study) contained lower levels of P. cibarius and Pseudoalteromonas group issachenkonii, but higher levels of $G$. arilaitensis, V. litoralis, M. ostreae, and Psychrobacter group nivimaris.

\section{DISCUSSION}

One key feature of Époisses cheese is the type of coagulation, which is mostly of the lactic type. Coagulation takes place for 16 to $24 \mathrm{~h}$, and the $\mathrm{pH}$ at the beginning of molding is about 4.5. Mesophilic starter cultures are used for the manufacturing of this cheese variety, which explains the dominance of lactococci at the beginning of ripening. Interestingly, in addition to $L$. lactis, there was a high level of $L$. chungangensis or L. laudensis. Little is known about the presence and activity of these species in cheeses, but there are at least 3 publications in which their occurrence in cheeses has been reported (Masoud et al., 2012; Frétin et al., 2018; Park et al., 2019). It would be interesting to investigate their potential functional properties relevant for cheese manufacturing.

Debaryomyces hansenii was the predominant yeast at the beginning of ripening. This can be explained by its deliberate inoculation at the beginning of manufacturing, its acid- and salt-tolerance, and its ability to grow at 5 to $10^{\circ} \mathrm{C}$. In addition, lactose and lactate, which constitute 2 important energy compounds for this species (Fröhlich-Wyder et al., 2019), were present at the beginning of ripening. Debaryomyces hansenii reached about $3 \times 10^{8} \mathrm{cfu} / \mathrm{cm}^{2}$ in the Époisses cheeses, which represents a high level considering that yeasts typically reach around $10^{6}$ to $10^{8} \mathrm{cfu} / \mathrm{cm}^{2}$ in smearripened cheeses (Cogan et al., 2014; Fröhlich-Wyder et al., 2019). Smear-ripened cheeses are also referred to as "bacterial smear surface-ripened cheeses" because bacteria are considered to be the dominant microorganisms in these cheeses typically representing 10 to 100 times higher colony counts than yeasts (Corsetti et al., 2001; Cogan et al., 2014). This trend was not observed for the Époisses cheeses investigated in the present study because the maximum count of surface bacteria was approximately the same as for the yeasts.

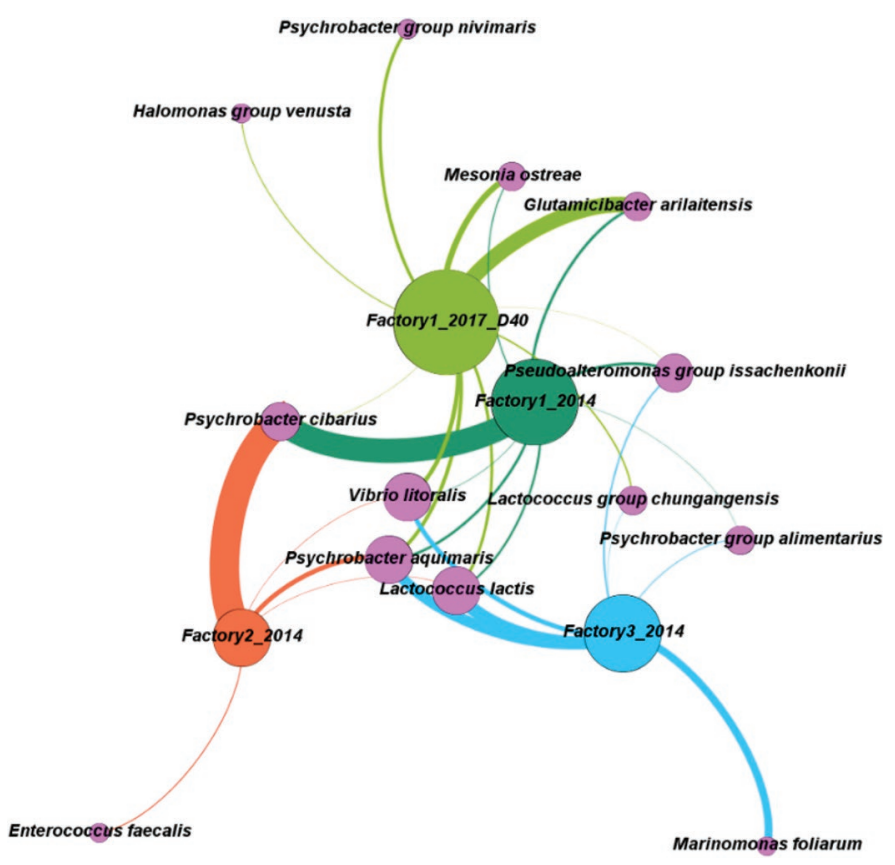

Figure 6. Operational taxonomic unit (OTU)-sample network summarizing the relationships between bacterial species of the cheese rinds from the present study at d 40 (sampled in 2017) and of cheeses sampled in 2014 and that were manufactured by the same factory (factory 1) or by 2 other Époisses factories (factories 2 and 3). Nodes represent OTU and cheeses. Connection lines indicate the detection of OTU in the cheeses. Only lines corresponding to OTU detected at $>0.5 \%$ relative abundance in the cheese rinds (mean of 3 samples) are shown. For the cheese nodes, different colors (green, dark green, orange, and blue) are used to differentiate cheese factories, and sizes are proportional to bacterial richness (i.e., number of connected OTU nodes per sample). For OTU nodes, sizes are proportional to the number of occurrences in the cheese rinds. The thickness of the connecting edges is proportional to the relative abundance of the OTU in the considered cheese. 
One possible explanation could be the low $\mathrm{pH}$ of the Époisses curd at the beginning of ripening, which delays the growth of the acid-sensitive aerobic bacteria. At least until d 21, the increase in $\mathrm{pH}$ (the $\mathrm{pH}$ value was 5.7 at day 21) could be attributed to the activity of the yeasts because the level of aerobic bacteria was very low. The yeasts contributed to the increase in the $\mathrm{pH}$ of the cheese surface by transforming lactate to $\mathrm{CO}_{2}$ and also by producing ammonia from AA. Both colony counts and ITS amplicon-sequencing analyses revealed that growth of $G$. candidum occurred later than that of $D$. hansenii. This is probably the consequence of the high salt content of the cheese surface at the beginning of ripening due to the salting procedure with dry salt. This favored the growth of $D$. hansenii because it is more tolerant to salt than G. candidum (Boutrou and Gueguen, 2005). Interestingly, the fungal diversity in the Époisses cheeses was very low because no species other than the 2 inoculated fungi $D$. hansenii and $G$. candidum could be detected. This is consistent with a previous study in which Époisses cheeses from 3 different producers were analyzed by amplicon highthroughput sequencing (Dugat-Bony et al., 2016). Consequently, there is no evidence that adventitious fungal species are common in Époisses cheeses. Several factors may be involved in the large decrease of $D$. hansenii viability that occurred during the last stage of ripening and during storage at low temperatures. One of these is lactose or lactate exhaustion, which has been proposed to explain D. hansenii lysis in experimental cheeses (Leclercq-Perlat et al., 1999). Other possible factors are the lower oxygen supply after cheese packaging or the cheese washing solution, which is supplemented with increasing amounts of Marc de Bourgogne during the ripening process. It is possible that the decrease of $D$. hansenii viability is a key feature of Époisses cheese manufacturing because it may also correspond to proteolytic and lipolytic enzymes liberated in the cheese curd by cell lysis (Kumura et al., 2002).

The present study also highlights the absence or poor growth of some ripening culture components at the cheese surface, as already observed in other studies (Brennan et al., 2002; Feurer et al., 2004; Mounier et al., 2005, 2006; Goerges et al., 2008; Cogan et al., 2014). The inoculated B. aurantiacum and S. xylosus strains were outcompeted by adventitious strains belonging to other species. It is noteworthy that among the 18 bacterial OTU detected ( $0.05 \%$ relative abundance cutoff), 14 corresponded to gram-negative species. At the end of ripening, gram-negative species accounted for about $70 \%$ of the $16 \mathrm{~S}$ rRNA sequences, with the most abundant genera being Psychrobacter, Halomonas, Mesonia, and Vibrio. One possible factor explaining the dominance of gram-negative species is the high moisture level of the Époisses rinds due the humid atmosphere of the ripening rooms and to the frequent washings of the curds (1-3 washings per week). Indeed, in a previous study of the community composition of 137 cheese rinds (Wolfe et al., 2014), a positive correlation was observed between rind moisture and some Proteobacteria genera such as Psychrobacter and Vibrio, whereas there was a negative correlation for the gram-positive genera Brevibacterium and Staphylococcus, which corresponded to the inoculated genera that did not establish themselves at the surface of the Époisses cheeses. One interesting feature of the cheese samples investigated in the present study was the presence of $M$. ostreae. To our knowledge, Mesonia has never been identified in cheese, except in 1 of the 3 Époisses cheese brands analyzed in 2014 (Dugat-Bony et al., 2016), which was produced by the same manufacturing unit as in the present study. The presence of $M$. ostreae is thus a specific signature of the cheese manufacturing unit considered here. $\mathrm{Me}$ sonia strains have mostly been isolated from marine environments, including seawater and seaweed; they are considered to be salt-tolerant, and some members are able to form biofilms (Lee et al., 2012; Huan et al., 2019), which may constitute useful properties for the growth on the cheese surface. The cheeses from the present study were manufactured from pasteurized milk, which is why the presence of high levels of uninoculated gram-negative species probably resulted from the facility-specific "house" microbiota, and especially environment microorganisms that were present on processing surfaces, materials, and airflows (Bokulich et al., 2016). The large presence of these microorganisms in the final product also raises the question about the efficiency of the inoculated ripening cultures used for manufacturing Époisses cheeses. The function of these cultures is to outcompete undesired microorganisms and to generate the typical sensory properties. It may be considered that the design of more efficient cultures requires the presence of gram-negative strains belonging to the genera Psychrobacter, Halomonas, Vibrio, or Mesonia, which are currently not used as ripening culture components. Interestingly, most gram-negative bacteria of dairy origin investigated in a recent safety assessment study were considered to be safe (Imran et al., 2019). Interestingly, B. aurantiacum, the orangepigmented bacterium that is used to give an orange color to cheeses, represented only a very small minority of the bacterial population. Therefore, this species is probably not responsible for the typical color of Époisses cheeses. In addition, HPLC profiles of the carotenoids from Époisses cheese rinds revealed the presence of yellow carotenoids produced by yellow bacteria such as G. arilaitensis, but not of orange carotenoids (Galaup et al., 2007). Furthermore, several studies reported the 
absence or presence at only very low levels of Brevibacterium in some orange smear-ripened cheeses (Brennan et al., 2002; Feurer et al., 2004; Bockelmann et al., 2005; Bockelmann, 2011; Delcenserie et al., 2014). It has also been suggested that several factors such as the proteolytic activity of smear bacteria may also be important for the typical color development of smearripened cheeses (Bockelmann and Hoppe-Seyler, 2001). Thus, it would be interesting to investigate the type and origin of the pigments that confer color to Époisses cheeses.

Another noteworthy observation was that during storage at $4^{\circ} \mathrm{C}$, between the packaging stage and the "bestbefore" date (d 90), there was a considerable change in bacterial composition of the Époisses cheeses. This may also occur in other cheese varieties, and it would be interesting to investigate microbial growth and activity during the storage of cheeses at low temperatures and its relationship to the shelf life of the product. In addition, the present study also revealed some changes in the microbial composition of cheeses produced by the same factory over a 3-yr interval. Whether this is common and possibly affects the sensory properties of the final product is not known, but a previous study reported major changes in the relative importance of the bacterial species present at the surface of Gubbeen cheeses manufactured in the same plant (Rea et al., 2007).

\section{CONCLUSIONS}

As observed at the surface of other smear-ripened cheeses, the acid-tolerant yeasts grow before the acidsensitive aerobic bacteria in Époisses cheese. However, in this cheese variety, the yeast Debaryomyces hansenii reached a high level during ripening, and there was subsequently a large decrease in its cell viability, which possibly affected the organoleptic properties of the final product. Most of the growth of the acid-sensitive bacteria occurred during the last week of ripening, and some changes in bacterial composition also occurred during postmanufacturing storage at $4^{\circ} \mathrm{C}$. The high moisture level probably favors gram-negative species, which are the dominant bacteria at the end of ripening. Because these species are able to outcompete part of the inoculated ripening bacteria, it might be interesting to devise ripening cultures containing typical gram-negative species present in this cheese variety and with previously assessed safety. The orange color of Époisses cheeses does not seem to be due to the presence of orangepigmented bacteria, and it would thus be interesting to investigate the type and origin of the pigments present in this cheese.

\section{ACKNOWLEDGMENTS}

This work has benefited from the high-throughput sequencing platform and expertise of GeT-PlaGE (http:/ /get.genotoul.fr). We are grateful to the INRAE Migale bioinformatics facility and to the Genotoul Bioinformatics Platform Toulouse Occitanie for providing help, computing, and storage resources. We are grateful to Eric Dugat-Bony (UMR SayFood, Thiverval-Grignon, France) for his assistance in bioinformatic analyses, and to Anne-Sophie Sarthou (UMR SayFood) for her excellent technical assistance. The authors have not stated any conflicts of interest.

\section{REFERENCES}

Bastian, M., S. Heymann, and M. Jacomy. 2009. Gephi: An Open Source Software for Exploring and Manipulating Networks. Pages 361-362 in Int. AAAI Conf. Web Soc. Media Third Int. AAAI Conf. Weblogs Soc. Media. AAAI Publications.

Bockelmann, W., and T. Hoppe-Seyler. 2001. The surface flora of bacterial smear-ripened cheeses from cow's and goat's milk. Int. Dairy J. 11:307-314. https://doi.org/10.1016/S0958-6946(01)00060-7.

Bockelmann, W. 2011. Cheese: Smear-ripened cheeses. Pages 753-766 in Encyclopedia of Dairy Sciences. 2nd ed. J. W. Fuquay, ed. Academic Press. https://doi.org/https://doi.org/10.1016/B978-0-12 -374407-4.00089-3.

Bockelmann, W., K. P. Willems, H. Neve, and K. H. Heller. 2005. Cultures for the ripening of smear cheeses. Int. Dairy J. 15:719-732. https://doi.org/10.1016/j.idairyj.2004.08.022.

Bokulich, N. A., Z. T. Lewis, K. Boundy-Mills, and D. A. Mills. 2016. A new perspective on microbial landscapes within food production. Curr. Opin. Biotechnol. 37:182-189. https://doi.org/10 .1016/j.copbio.2015.12.008.

Boutrou, R., and M. Gueguen. 2005. Interests in Geotrichum candidum for cheese technology. Int. J. Food Microbiol. 102:1-20. https://doi .org/10.1016/j.ijfoodmicro.2004.12.028.

Brennan, N. M., A. C. Ward, T. P. Beresford, P. F. Fox, M. Goodfellow, and T. M. Cogan. 2002. Biodiversity of the bacterial flora on the surface of a smear cheese. Appl. Environ. Microbiol. 68:820830. https://doi.org/10.1128/AEM.68.2.820-830.2002.

Castellote, J., S. Fraud, F. Irlinger, D. Swennen, F. Fer, P. Bonnarme, and C. Monnet. 2015. Investigation of Geotrichum candidum gene expression during the ripening of Reblochon-type cheese by reverse transcription-quantitative PCR. Int. J. Food Microbiol. 194:54-61. https://doi.org/10.1016/j.ijfoodmicro.2014.11.009.

CNIEL. 2020. Dairy economy - edition 2020, Accessed Feb. 1, 2021. https://presse.filiere-laitiere.fr/assets/economie-laitieres-en -chiffres-edition-2020-fa05-ef05e.html?lang=fr.

Cogan, T. M., S. Goerges, R. Gelsomino, S. Larpin, M. Hohenegger, N. Bora, E. Jamet, M. C. Rea, J. Mounier, V. M., M. Guéguen, N. Desmasures, J. Swings, M. Goodfellow, A. C. Ward, H. Sebastiani, F. Irlinger, J. F. Chamba, R. Beduhn, and S. Scherer. 2014. Biodiversity of the surface microbial consortia from Limburger, Reblochon, Livarot, Tilsit, and Gubbeen cheeses. C. W. Donnelly, ed. ASM Press.

Corsetti, A., J. Rossi, and M. Gobbetti. 2001. Interactions between yeasts and bacteria in the smear surface-ripened cheeses. Int. J. Food Microbiol. 69:1-10. https://doi.org/10.1016/S0168 -1605(01)00567-0.

De Filippis, F., E. Parente, and D. Ercolini. 2018. Recent past, present, and future of the food microbiome. Annu. Rev. Food Sci. Technol. 9:589-608. https://doi.org/10.1146/annurev-food-030117 -012312 .

Delcenserie, V., B. Taminiau, L. Delhalle, C. Nezer, P. Doyen, S. Crevecoeur, D. Roussey, N. Korsak, and G. Daube. 2014. Micro- 
biota characterization of a Belgian protected designation of origin cheese, Herve cheese, using metagenomic analysis. J. Dairy Sci. 97:6046-6056.

Dugat-Bony, E., L. Garnier, J. Denonfoux, S. Ferreira, A.-S. Sarthou, P. Bonnarme, and F. Irlinger. 2016. Highlighting the microbial diversity of 12 French cheese varieties. Int. J. Food Microbiol. 238:265-273. https://doi.org/10.1016/j.ijfoodmicro.2016.09.026.

Dugat-Bony, E., C. Straub, A. Teissandier, D. Onésime, V. Loux, C. Monnet, F. Irlinger, S. Landaud, M.-N. Leclercq-Perlat, P. Bento, S. Fraud, J.-F. Gibrat, J. Aubert, F. Fer, E. Guédon, N. Pons, S. Kennedy, J.-M. Beckerich, D. Swennen, and P. Bonnarme. 2015. Overview of a surface-ripened cheese community functioning by meta-omics analyses. PLoS One 10:e124360. https://doi.org/10 .1371/journal.pone.0124360.

Escudié, F., L. Auer, M. Bernard, M. Mariadassou, L. Cauquil, K. Vidal, S. Maman, G. Hernandez-Raquet, S. Combes, and G. Pascal. 2018. FROGS: Find, Rapidly, OTUs with Galaxy Solution. Bioinformatics 34:1287-1294. https://doi.org/10.1093/bioinformatics/ btx791.

Feurer, C., T. Vallaeys, G. Corrieu, and F. Irlinger. 2004. Does smearing inoculum reflect the bacterial composition of the smear at the end of the ripening of a french soft, red-smear cheese? J. Dairy Sci. 87:3189-3197. https://doi.org/10.3168/jds.S0022-0302(04)73454 -2 .

Frétin, M., B. Martin, E. Rifa, V.-M. Isabelle, D. Pomiès, A. Ferlay, M.-C. Montel, and C. Delbès. 2018. Bacterial community assembly from cow teat skin to ripened cheeses is influenced by grazing systems. Sci. Rep. 8:1-11.

Fröhlich-Wyder, M.-T., E. Arias-Roth, and E. Jakob. 2019. Cheese yeasts. Yeast 36:129-141.

Galaup, P., A. Gautier, Y. Piriou, A. de Villeblanche, A. Valla, and L. Dufossé. 2007. First pigment fingerprints from the rind of French PDO red-smear ripened soft cheeses Epoisses, Mont d'Or and Maroilles. Innov. Food Sci. Emerg. Technol. 8:373-378. https://doi .org/10.1016/j.ifset.2007.03.017.

Goerges, S., J. Mounier, M. C. Rea, R. Gelsomino, V. Heise, R. Beduhn, T. M. Cogan, M. Vancanneyt, and S. Scherer. 2008. Commercial ripening starter microorganisms inoculated into cheese milk do not successfully establish themselves in the resident microbial ripening consortia of a south German red smear cheese. Appl. Environ. Microbiol. 74:2210-2217. https://doi.org/10.1128/AEM.01663-07.

Huan, R., J. Huang, D. Liu, M. Wang, C. Liu, Y. Zhang, C. Yi, D. Xiao, and H. He. 2019. Genome Sequencing of Mesonia algae K4-1 reveals its adaptation to the Arctic ocean. Front. Microbiol. 10:2812.

Imran, M., N. Desmasures, M. Coton, E. Coton, A. Le Flèche-Matéos, F. Irlinger, C. Delbès-Paus, V. Stahl, M.-C. Montel, and J.-P. Vernoux. 2019. Safety assessment of Gram-negative bacteria associated with traditional French cheeses. Food Microbiol. 79:1-10.

ISO. 2016. 8968-4: Milk and milk products-Determination of nitrogen content-Part 4: Determination of protein and non-protein nitrogen content and true protein content calculation (Reference method). https://standards.iteh.ai/catalog/standards/sist/ ad8f8310-0260-4b67-a560-9931549a4e11/iso-8968-4-2016.

Jonnala, B. R. Y., P. L. H. McSweeney, J. J. Sheehan, and P. D. Cotter. 2018. Sequencing of the cheese microbiome and its relevance to industry. Front. Microbiol. 9. https://doi.org/10.3389/fmicb.2018 .01020 .

Kim, O.-S., Y.-J. Cho, K. Lee, S.-H. Yoon, M. Kim, H. Na, S.-C. Park, Y. S. Jeon, J.-H. Lee, H. Yi, S. Won, and J. Chun. 2012. Introducing EzTaxon-e: A prokaryotic 16S rRNA gene sequence database with phylotypes that represent uncultured species. Int. J. Syst. Evol. Microbiol. 62:716-721. https://doi.org/10.1099/ijs .0.038075-0.

Kumura, H., K. Takagaki, T. Sone, M. Tsukahara, T. Tanaka, and K. Shimazaki. 2002. Casein digestion by Debaryomyces hansenii isolated from cheese. Biosci. Biotechnol. Biochem. 66:1370-1373. https://doi.org/10.1271/bbb.66.1370.
Leclercq-Perlat, M. N., A. Oumer, J. L. Bergere, H. E. Spinnler, and G. Corrieu. 1999. Growth of Debaryomyces hansenii on a bacterial surface-ripened soft cheese. J. Dairy Res. 66:271-281. https://doi .org/10.1017/S0022029999003362.

Leclercq-Perlat, M. N., D. Picque, S. T. Martin del Campo Barba, and C. Monnet. 2013. Dynamics of Penicillium camemberti growth quantified by real-time PCR on Camembert-type cheeses under different conditions of temperature and relative humidity. J. Dairy Sci. 96:4031-4040. https://doi.org/10.3168/jds.2012-6372.

Lee, S.-Y., M.-H. Lee, and J.-H. Yoon. 2012. Mesonia ostreae sp. nov., isolated from seawater of an oyster farm, and emended description of the genus Mesonia. Int. J. Syst. Evol. Microbiol. 62:1804-1808.

LEGIFRANCE. 2010. Decree no. 2010-1092 relating to the Protected Designation of Origin (PDO) "Epoisses." Accessed Feb. 1, 2021. https://www.legifrance.gouv.fr/loda/id/ JORFTEXT000022825025/2021-02-01/.

Magoč, T., and S. L. Salzberg. 2011. FLASH: Fast length adjustment of short reads to improve genome assemblies. Bioinformatics 27:2957-63. https://doi.org/10.1093/bioinformatics/btr507.

Mahé, F., T. Rognes, C. Quince, C. de Vargas, and M. Dunthorn. 2014. Swarm: Robust and fast clustering method for ampliconbased studies. PeerJ 2:e593.

Masoud, W., F. K. Vogensen, S. Lillevang, W. Abu Al-Soud, S. J. Sørensen, and M. Jakobsen . 2012. The fate of indigenous microbiota, starter cultures, Escherichia coli, Listeria innocua and Staphylococcus aureus in Danish raw milk and cheeses determined by pyrosequencing and quantitative real time (qRT)-PCR. Int. J. Food Microbiol. 153:192-202. https://doi.org/10.1016/j.ijfoodmicro .2011.11.014.

McMurdie, P. J., and S. Holmes. 2013. phyloseq: an R package for reproducible interactive analysis and graphics of microbiome census data. PLoS One 8:e61217.

Mounier, J., R. Gelsomino, S. Goerges, M. Vancanneyt, K. Vandemeulebroecke, B. Hoste, S. Scherer, J. Swings, G. F. Fitzgerald, and T. M. Cogan. 2005. Surface microflora of four smear-ripened cheeses. Appl. Environ. Microbiol. 71:6489-6500. https://doi.org/ 10.1128/AEM.71.11.6489-6500.2005.

Mounier, J., S. Goerges, R. Gelsomino, M. Vancanneyt, K. Vandemeulebroecke, B. Hoste, N. M. Brennan, S. Scherer, J. Swings, G. F. Fitzgerald, and T. M. Cogan. 2006. Sources of the adventitious microflora of a smear-ripened cheese. J. Appl. Microbiol. 101:668 681. https://doi.org/10.1111/j.1365-2672.2006.02922.x.

Nilsson, R. H., K.-H. Larsson, A. F. S. Taylor, J. Bengtsson-Palme, T. S. Jeppesen, D. Schigel, P. Kennedy, K. Picard, F. O. Glöckner, L. Tedersoo, I. Saar, U. Kõljalg, and K. Abarenkov. 2019. The UNITE database for molecular identification of fungi: Handling dark taxa and parallel taxonomic classifications. Nucleic Acids Res. 47:D259-D264.

Park, W., J. Yoo, S. Oh, J. Ham, S. Jeong, and Y. Kim. 2019. Microbiological characteristics of Gouda cheese manufactured with pasteurized and raw milk during ripening using next generation sequencing. Food Sci. Anim. Resour. 39:585-600.

Rea, M. C., S. Gorges, R. Gelsomino, N. M. Brennan, J. Mounier, M. Vancanneyt, S. Scherer, J. Swings, and T. M. Cogan. 2007. Stability of the biodiversity of the surface consortia of Gubbeen, a red-smear cheese. J. Dairy Sci. 90:2200-2210. https://doi.org/10 $.3168 /$ jds.2006-377.

Wolfe, B. E., J. E. Button, M. Santarelli, and R. J. Dutton. 2014. Cheese rind communities provide tractable systems for in situ and in vitro studies of microbial diversity. Cell 158:422-433. https:// doi.org/10.1016/j.cell.2014.05.041.

\section{ORCIDS}

F. Irlinger (ㅇ https://orcid.org/0000-0001-7883-7589

C. Monnet ๑ https://orcid.org/0000-0003-2647-272X 